\title{
Steiner Minimal Trees for Regular Polygons
}

\author{
D. Z. Du, ${ }^{1}$ F. K. Hwang, ${ }^{2}$ and J. F. Weng ${ }^{3}$ \\ ' University of California, Santa Barbara, California, USA, also from Academia Sinica, Beijing, China \\ ${ }^{2}$ AT\&T Bell Laboratories, Murray Hill, NJ 07974, USA \\ ${ }^{3}$ Baoshan General Iron and Steel Works, Shanghai, China
}

\begin{abstract}
Fifty years ago Jarnik and Kössler showed that a Steiner minimal tree for the vertices of a regular $n$-gon contains Steiner points for $3 \leq n \leq 5$ and contains no Steiner point for $n=6$ and $n \geq 13$. We complete the story by showing that the case for $7 \leq n \leq 12$ is the same as $n \geq 13$. We also show that the set of $n$ equally spaced points yields the longest Steiner minimal tree among all sets of $n$ cocircular points on a given circle.
\end{abstract}

\section{Introduction}

A Steiner minimal tree (SMT) for a set of points $P$ in the plane is a shortest network interconnecting $P$. The construction of an SMT for a general set $P$ is known [7] to be an NP-complete problem. Recently, SMTs have been constructed for special point sets $\boldsymbol{P}$ such as ladders [1], splitting trees [9], zigzag lines [5], cocircular points [6], and bar waves [4]. However, a special class of sets for which the study of SMTs was started a half century back has remained an unsolved problem. Let $\boldsymbol{A}_{n}$ denote the set of vertices of a regular $\boldsymbol{n}$-gon. The SMT problem for $A_{n}$ was first studied by Jarnik and Kössler [10] in 1934. They obtained SMTs for $n \leq 6$ and also proved a beautiful theorem which says that for $n \geq 13$ an SMT can be obtained by deleting an edge from the perimeter of the regular $n$-gon. Since an SMT can also be obtained in this manner for $n=6$, an obvious conjecture is that an SMT can be so obtained for all $n \geq 6$. Kotzig [11] discussed some properties of the angles of an SMT for $n \leq 8$. In this article we will prove this conjecture in its entirety as our Theorem 1.

Theorem 1. The perimeter of a regular n-gon minus any side is an SMT for $A_{n}$ for $n \geq 6$. 
We also prove

Theorem 2. For any $n$ cocircular points on a given circle, the set of $n$ equally spaced points yields a longest SMT.

\section{The Case $n \geq 11$}

In this section we show that some recent results on the Steiner ratio (to be defined shortly) can be used to dispose of the conjecture for all $n \geq 11$.

A minimal spanning tree (MST) for a set of points $P$ is a shortest tree interconnecting $P$ such that the vertex-set of the tree is $P$. The Steiner ratio $\rho$ is defined as

$$
\inf _{P} \frac{\text { length of an SMT for } P}{\text { length of an MST for } P}
$$

Gilbert and Pollak [8] cojectured that $\rho=\sqrt{3} / 2$ while Du and Hwang [3] proved that $\rho \geq 0.8$. Recently, Chung and Graham [2] announced a proof that $\rho \geq 0.8241$. The Steiner ratio was surprisingly used in [6] to prove a result about SMTs for cocircular points, via the following lemma:

Lemma 0. Suppose that an n-gon circumscribed in a unit circle has at most one side longer than $m$ with

$$
m=\min \left\{\left[\alpha \beta+\sqrt{\left.\alpha^{2}+\left(1-\beta^{2}\right) / 4\right]} /\left(\alpha^{2}+\frac{1}{4}\right), \gamma\right\},\right.
$$

where

$$
\begin{aligned}
& \alpha=\sqrt{3}+1-1 /(2 \bar{\rho}), \\
& \beta=1-(1-\bar{\rho}) \pi / \bar{\rho}
\end{aligned}
$$

( $\bar{\rho}$ is a lower bound for $\rho$ ) and

$$
\gamma=2(\sqrt{3}+1) /\left[(\sqrt{3}+1)^{2}+\frac{1}{4}\right]=0.708 \ldots
$$

Then its MST (which is the perimeter of the n-gon minus the logest side) is also its SMT.

Set $\bar{\rho}=\mathbf{0 . 8 2 4}$. We obtain $m>0.6034$. On the other hand, the length of a side of the regular $n$-gon

$$
l_{n}=\sqrt{2\left(1-\cos \frac{2 \pi}{n}\right)}=2 \sin \frac{\pi}{n}
$$

is monotone decreasing in $n$ for $n \geq 3$. Furthermore,

$$
l_{n} \leq l_{11}<0.5635<0.6034<m \text { for } n \leq 11 \text {. }
$$


By Lemma 0 we obtain

Theorem 1. The MST of a regular $n$-gon is also its $S M T$ for $n \geq 11$.

\section{Some Facts About SMTs}

Consider any tree $T$ interconnecting a set of points $P=\left\{p_{1}, \ldots, p_{n}\right\}$. We will refer to the $p_{i}$ 's as the regular points and any other points in $T$ as Steiner points. $T$ is called a Steiner tree if all subtending angles are at least $120^{\circ}$ and each Steiner point has three incident edges (this implies that the subtending angles are exactly $120^{\circ}$ for a Steiner point). It is well known [8] that a Steiner tree for $n$ points has at most $n-2$ Steiner points and is called a full Steiner tree if it has $n-2$ points. It is also well known [8] that an SMT must be a Steiner tree and can always be decomposed into subtrees which are full Steiner trees. Finally, it is well known [8] that an SMT always lies within the convex hull of $P$.

A topology of a Steiner tree $T$ is a specification of all edges in $T$. A Steiner tree for a given topology either exists uniquely or does not exist. When a full Steiner tree with a given topology exists, Melzak [12] gave a recursive construction for it which also yields a line segment, which we call the axis, whose length equals that of the Steiner tree.

Let $C$ denote a unit circle with center $o$. Let $R_{n}$ denote a regular $n$-gon inscribed in $C$ with vertex set $A_{n}=\left\{a_{1}, \ldots, a_{n}\right\}$. Throughout the paper we denote the line segment between two points $x$ and $y$ by $[x, y]$ and its length by $(x y)$.

Lemma 1. Let $T$ be an SMT for $R_{n}$. Then we may assume that no Steiner point $s$ of $T$ can have an incident edge as long as $l_{n}$.

Proof. Suppose to the contrary that $l$ is such an edge. Delete $l$ and decompose $T$ into two subtrees. Then there must exist a $j$ such that $a_{j}$ and $a_{j+1}$ are not in the same subtree. Connect $a_{j}, a_{j+1}$ and we obtain an interconnecting tree not longer than $T$.

Lemma 2. Let $C$ be a unit circle with center o. Let $p, q$ be two points such that $(p o) \geq 1 \geq(q o)$ and $\Varangle o q p \leq 60^{\circ}$ (see Fig. 1). Then $(p q) \geq(p o)$.

Proof. In $\triangle o p q, \Varangle q p o \leq \Varangle o q p \leq 60^{\circ}$ since $(p o) \geq(q o)$. Hence $\Varangle p o q \geq 60^{\circ} \geq \Varangle o q p$. It follows $(p q) \geq(p o)$.

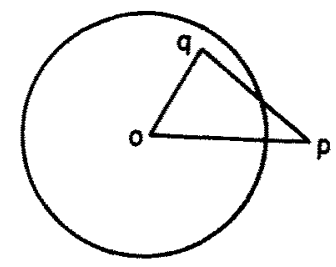

Fig. 1. $(p q)>(p o)$. 
The following Lemma is not directly related to SMTs but often facilitates an argument that a certain topology does not exist.

Lemma 3. Let $C A_{1} \ldots A_{m} D$ be a polygon lying within another polygon $C B_{1} \ldots B_{n} D$. Then

$$
\sum_{i=1}^{m} \not A_{i}-\sum_{i=1}^{n} \Varangle B_{i} \geq(m-n) 180^{\circ}
$$

Proof. Using the fact that an $n$-gon has total inner degrees $(n-2) 180^{\circ}$ and the fact that $\Varangle A_{1} C D \leq \Varangle B_{1} C D$ and $\Varangle C D A_{m} \leq \Varangle C D B_{n}$.

A path $a_{i} s_{1} \ldots s_{m} a_{j}$ in an SMT $T$ is called a Steiner path if $s_{1}, \ldots, s_{n}$, are all Steiner points and $\Varangle s_{i}=120$ for $i=1, \ldots, m$ in the $(m+2)$-gon $a_{i} s_{1} \ldots s_{m} a_{j}$.

Lemma 4. Suppose that $T$ is an $S M T$ for $R_{n}$. Let $P=a_{i} s_{1} s_{2} \ldots s_{m} a_{j}$ be a Steiner path.

(i) $m \leq 3$. There are no regular points between $a_{i}$ and $a_{j}$.

(ii) $m=4$. There is at most one regular point between $a_{i}$ and $a_{j}$ but none if $n \leq 9$. No such $P$ can exist for $n \leq 6$.

(iii) $m=5$. No such $P$ can exist for $n \leq 11$.

(iv) $m \geq 6$. No such $P$ can exist.

Proof. It is easily verified that $\left(a_{i} a_{j}\right)<2 l_{n}$ for all $m$. Thus at most one regular point can exist between $a_{i}$ and $a_{j}$.

$m=1$ or 2 . Suppose to the contrary that $a_{i}$ and $a_{j}$ are not adjacent. Then $n \geq 4$. Let $a_{k}$ be the regular point between $a_{i}$ and $a_{j}$. For $m=1$ consider the quadrilateral $a_{i} s_{1} a_{j} a_{k}$. We have

$$
\Varangle a_{i}+\not a_{j}=360^{\circ}-\not s_{1}-\not a_{k} \leq 360^{\circ}-120^{\circ}-90^{\circ}=150^{\circ} .
$$

For $m=2$ consider the pentagon $a_{i} s_{1} s_{2} a_{j} a_{k}$. We have

$$
\Varangle a_{i}+\Varangle a_{j}=540^{\circ}-\Varangle s_{1}-\Varangle s_{2}-\Varangle a_{k} \leq 540^{\circ}-120^{\circ}-120^{\circ}-90^{\circ}=210^{\circ} \text {. }
$$

Hence either $\Varangle a_{i}$ or $\Varangle a_{j}$ is less than $120^{\circ}$. Suppose that $\Varangle a_{i}<120^{\circ}$. Then $a_{k}$ is connected to $a_{j}$ in $T$. Let $T^{\prime}$ be obtained by substituting $\left[a_{k}, a_{i}\right]$ for $\left[a_{k}, a_{j}\right]$ in $T$. Then $T^{\prime}$ and $T$ have the same length. Yet $T^{\prime}$ cannot be optimal since $\Varangle a_{k} a_{i} s_{1}<120^{\circ}$, a contradiction to the optimality of $T$.

$m=3$. We have $n \geq m+2=5$. If $n=5$, then there is not other Steiner point and each of $s_{1}, s_{2}$, and $s_{3}$ must connect a distinct regular point outside of the pentagon $a_{i} s_{1} s_{2} s_{3} a_{j}$. Thus there is no more regular point to fill between $a_{i}$ and $a_{j}$. Therefore we may assume that $n \geq 6$. 
Suppose to the contrary that $a_{k}$ exists between $a_{i}$ and $a_{j}$. Consider the hexagon $a_{i} s_{1} s_{2} s_{3} a_{j} a_{k}$. Note that all angles except $\Varangle a_{i}$ and $\Varangle a_{j}$ are at least $120^{\circ}$. Hence either the hexagon is regular, or at least one of $\Varangle a_{i}$ and $\Varangle a_{j}<120^{\circ}$. The former case is impossible since we do not allow $\left(s_{1} s_{2}\right)=\left(a_{k} a_{i}\right)=l_{n}$. The latter case is also impossible by analogous argument as used in the case $m=1$ or 2 .

$m=4$. $n \geq m+2=6$. If $n=6$, the hexagon $a_{i} s_{1} s_{2} s_{3} s_{4} a_{j}$ has internal degree less than $720^{\circ}$, an absurdity. For $n \geq 7$ suppose that $a_{k}$ exists between $a_{i}$ and $a_{j}$. For $n \leq 9$, the heptagon $a_{i} s_{1} s_{2} s_{3} s_{4} a_{j} a_{k}$ has internal degrees less than $900^{\circ}$, an absurdity.

$m=5$. Consider the heptagon $a_{1} s_{1} s_{2} s_{3} s_{4} s_{5} a_{j}$,

$$
\Varangle a_{i}+\Varangle a_{j}=900^{\circ}-5 \cdot 120^{\circ}=300^{\circ}>\measuredangle a_{i-1} a_{i} a_{i+1}+\Varangle a_{j-1} a_{j} a_{j+1} \quad \text { for } n \leq 11 \text {, }
$$

an absurdity.

$m \geq 6$. Consider the $(m+2)$-gon $a_{i} s_{1} \ldots s_{m} a_{j}$,

$$
\Varangle a_{i}+\Varangle a_{j}=m \cdot 180^{\circ}-m \cdot 120^{\circ}=m \cdot 60^{\circ} \geq 360^{\circ} \text { for } m \geq 6 \text {, an absurdity. }
$$

Lemma 5. Let $T$ be an $S M T$ for $A_{n}, n \leq 10$, with a Steiner point $s$. Then $T$ must be full.

Proof. Let $T^{\prime} \subseteq T$ be a full Steiner tree containing $s$. Then $T^{\prime}$ partitions the unit circle into convex regions each bounded by a Steiner path and an arc. By Lemma 4 such an arc can contain at most one additional regular point. In fact, the only case in which an additional regular point may exist is when $n=10$ and the Steiner path bounding the region has $m=4$. We now show that even for this case no additional regular point can exist on the arc, i.e., $T$ is a full SMT.

Suppose to the contrary that a regular point $a_{k}$ exists on the arc $a_{i} a_{j}$ (see Fig. 2). In the heptagon $a_{i} s_{1} s_{2} s_{3} s_{4} a_{j} a_{k}$

$$
\not a_{i}+\not a_{j}=900^{\circ}-4 \times 120^{\circ}-144^{\circ}=276^{\circ} .
$$

Therefore

$$
\Varangle s_{1} a_{i} a_{i+1}+\Varangle a_{j-1} a_{j} s_{4}=2 \times 144^{\circ}-276^{\circ}=12^{\circ}
$$

and

$$
\min \left\{\left(s_{1} a_{i+1}\right),\left(s_{4} a_{j-1}\right) \geq l_{n} \sin 48^{\circ} / \sin 120^{\circ}>0.858 l_{n}\right.
$$

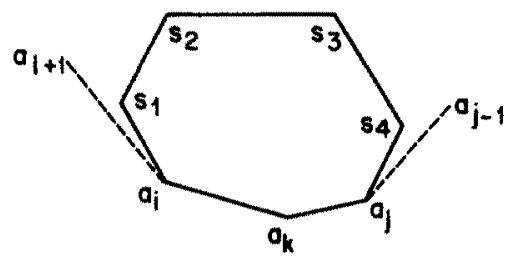

Fig. 2. A Steiner path with $m=4$ and a regular point. 
(it is easily verified that $\left(s_{1} a_{i+1}\right)$ will be greater if $s_{1}$ connects $a_{i+1}$ through another Steiner point). Now it is a simple matter to show that $\left(s_{1} s_{4}\right)>2 l_{n}$ which implies that one of $\left(s_{1} s_{2}\right),\left(s_{2} s_{3}\right)$, and $\left(s_{3} s_{4}\right)>l_{n}$, a contradiction to Lemma 1 .

Define $D$ to be the diameter (of the unit circle $c$ ) $\perp\left[a_{1}, a_{n}\right]$.

Lemma 6. Suppose that a topology is symmetric with respect to an edge e. Then the Steiner tree it yields is symmetric with respect to $D$ with e overlapping $D$ for odd $n$ and $e \perp D$ for even $n$. Suppose that a topology is symmetric with respect to a point p. Then the Steiner tree it yields is symmetric with respect to the center $o$ with $p$ being 0 .

Proof. Clear from Melzak's construction for SMT.

\section{Proof of Theorem 1 for $8 \geq n \geq 10$}

Suppose that $T$ is an SMT for $A_{n}$ with a Steiner point. By Lemma 5 we may assume that $T$ is full. Let $d$ be a point of $T$ closest to the center $o$ and let $d$ lie on the edge $e$. Let $q$ be an endpoint of $e$. Partition $T$ into two trees $T_{1}$ and $T_{2}$ at $q$ and without loss of generality assume that $T_{1}$ contains $e$ and the $k$ regular points $\left\{a_{1}, a_{2}, \ldots, a_{k}\right\}$. By Melzak's construction of the full Steiner tree, there exists a line segment $[p, q]$ which is the axis of $T_{1}$ and overlaps $e$. Our goal is to show that for certain $n T_{1}$ cannot exist by proving $(p q)>k l_{n}$, so $T_{1}$ can be replaced by the path $a_{1} a_{2} \ldots a_{k}$ and some suitable $\left[a_{j}, a_{j+1}\right]$ to obtain a shorter connecting tree. However, since $(p o)$ is much easier to compute than $(p q)$, we will prove $p o>k l_{n}$ instead and use Lemma 2 to justify the replacement. One condition of Lemma 2 is that $\Varangle p q o \leq 60$. The following lemma will take care of that condition.

Lemma 7. $\Varangle d q o>60^{\circ}$.

Proof. Let $e^{\prime}$ be a second edge of $T$ at $q$ such that $o$ lies in the $120^{\circ}$ angle enclosed by $e$ and $e^{\prime}$ (possibly their extensions). Let (od ) be the distance from $o$ to $e^{\prime}$. Since $(o d) \leq\left(o d^{\prime}\right), \Varangle d q o \leq \Varangle d^{\prime} q o$. But $\Varangle d q o+\not d^{\prime} q o=120^{\circ}$. Hence $4 d q o \leq 60^{\circ}$.

Lemma 8. $k \neq 1$ for $n>6$.

Proof. From Lemmas $7 \Varangle a_{1} q o \leq 60^{\circ}$. From Lemma 2

$$
\left(a_{1} q\right) \geq\left(a_{1} o\right)=1>l_{n} \quad \text { for } n>6 .
$$

Lemma 9. $k \neq 2$ for $8 \leq n \leq 10$.

Proof. Suppose to the contrary that $k=2$. Let $p a_{1} a_{2}$ be a regular triangle with $p$ outside of the unit circle. Then by Melzak's construction $[p, q]$ is the axis of 
$T_{1}$. We now prove that $(p o)>2 l_{n}$, or $\left((p o) / l_{n}\right)^{2}>4$ :

$$
\begin{aligned}
(p o)^{2} & =\left(a_{1} o\right)^{2}+\left(a_{1} p\right)^{2}-2\left(a_{1} o\right)\left(a_{1} p\right) \cos 4 o a_{1} p \\
& =1+l_{n}^{2}-2 l_{n} \cos \left(90^{\circ}-180^{\circ} / n+60^{\circ}\right) \\
& =1+l_{n}^{2}+\sqrt{3} l_{n} \cos \left(180^{\circ} / n\right)-l_{n} \sin \left(180^{\circ} / n\right) \\
& =1+l_{n}^{2}+\sqrt{3} l_{n} \sqrt{1-l_{n}^{2} / 4}-l_{n}^{2} / 2 \\
& =1+l_{n}^{2} / 2+\sqrt{3} l_{n} \sqrt{1-l_{n}^{2} / 4} .
\end{aligned}
$$

Clearly, $(p o)^{2} / l_{n}^{2}$ is monotone decreasing in $l_{n}^{2}$. For $8 \leq n \leq 10 l_{n}^{2}$ is largest for $n=8$ and $l_{8}^{2}=2\left(1-\cos 45^{\circ}\right)=2-\sqrt{2}$. Now

$$
\left(\frac{(p o)}{l_{8}}\right)^{2}=\frac{1}{2-\sqrt{2}}+\frac{1}{2}+\sqrt{3}-\frac{1}{4} \geq 4.297>4 \text {. }
$$

Lemma 10. $k \neq 3$ for $8 \leq n \leq 10$.

Proof. Suppose to the contrary that $k=3$. Let $a_{3} a_{2} p_{1}$ and $p_{1} a_{1} p_{2}$ be regular triangles such that $p_{1}$ and $o$ are on different sides of $\left[a_{2}, a_{3}\right]$, and $p_{2}$ and $o$ are on different sides of $\left[p_{1} a_{1}\right]$ (see Fig. 3). We now prove that $\left(p_{2} o\right)>3 l_{n}$ or $\left.\left(p_{2} o\right) / l_{n}\right)^{2}>9$. Note that

Now

$$
\left(p_{2} o\right)^{2}=\left(p_{1} o\right)^{2}+\left(p_{1} p_{2}\right)^{2}-2\left(p_{1} o\right)\left(p_{1} p_{2}\right) \cos \Varangle o p_{1} p_{2}
$$

$$
\begin{aligned}
\left(p_{1} p_{2}\right)=\left(p_{1} a_{1}\right) & =\left[\left(a_{1} a_{2}\right)^{2}+\left(p_{1} a_{2}\right)^{2}-2\left(a_{1} a_{2}\right)\left(p_{1} a_{2}\right) \cos \Varangle a_{1} a_{2} p_{1}\right]^{1 / 2} \\
& =2 l_{n}\left[\left(1-\cos \Varangle a_{1} a_{2} p_{1}\right) / 2\right]^{1 / 2} \\
& =2 l_{n} \sin \left(\Varangle a_{1} a_{2} p_{1} / 2\right) \\
& =2 l_{n} \sin \left(60^{\circ}+180^{\circ} / n\right) .
\end{aligned}
$$

Hence

$$
\left(p_{1} p_{2}\right) / l_{n}=2 \sin \left(60^{\circ}+180^{\circ} / n\right) \geq 2 \sin \left(60^{\circ}+18^{\circ}\right)>1.956
$$

and $\left(p_{1} p_{2}\right)^{2} / l_{n}^{2}>3.827$ for $n \leq 10$. Furthermore,

$$
\begin{aligned}
\Varangle o p_{1} p_{2} & =\Varangle o p_{1} a_{2}+\Varangle a_{2} p_{1} a_{1}+\Varangle a_{1} p_{1} p_{2} \\
& =30^{\circ}+30^{\circ}-180^{\circ} / n+60^{\circ}=120^{\circ}-180^{\circ} / n
\end{aligned}
$$

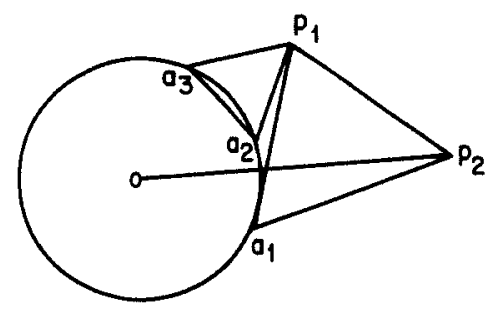

Fig. 3. $\left(p_{2} 0\right)>3 l_{n}$ for $k=3$. 
and

$$
-2 \cos \measuredangle o p_{1} p_{2}=2 \cos \left(60^{\circ}+180^{\circ} / n\right),
$$

which is clearly monotone increasing in $n$ for $n \geq 6$. Therefore, for $8 \leq n \leq 10$

$$
-2 \cos \measuredangle o p_{1} p_{2} \geq 2 \cos 82.5^{\circ}=0.261 \text {. }
$$

Therefore, for $8 \leq n \leq 10$ we have

$$
\left(\frac{p_{2} o}{l_{n}}\right)^{2} \geq 4.297+3.827+(0.261) \sqrt{(4.297)(3.827)}=9.182>9 \text {. }
$$

Lemma 11. $k \neq 4$ for $8 \leq n \leq 10$.

Proof. Suppose to the contrary that $k=4$. There are three nonisomorphic topologies for $T_{1}$ which we will call topologies 4, 5, and 6 and their Melzak's constructions are shown in Figs. 4-6, respectively. We show that the axis of $T_{1}$ is too long for all three topologies.

Topology 4 (Fig. 4)

As shown in the proof of Lemma 10,

$$
\begin{aligned}
\left(a_{2} p_{2}\right) & =2 l_{n} \sin \left(60^{\circ}+\frac{180^{\circ}}{n}\right), \\
{ }_{4} a_{3} a_{2} p_{1} & =90^{\circ}-\left(60^{\circ}+\frac{180^{\circ}}{n}\right) \\
& =30^{\circ}-\frac{180^{\circ}}{n} .
\end{aligned}
$$

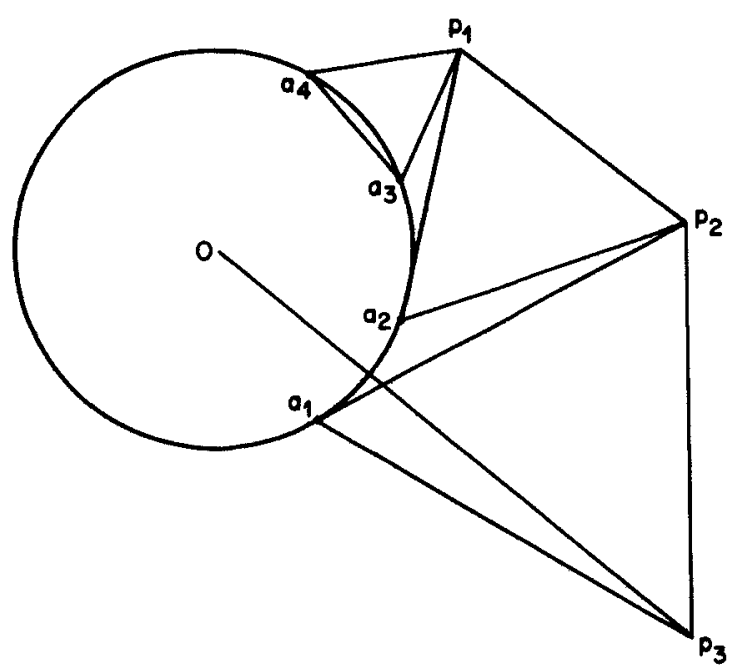

Fig. 4. Melzak's construction for topology 4. 
Therefore,

$$
\begin{aligned}
\Varangle a_{1} a_{2} p_{2} & =90^{\circ}+\frac{540^{\circ}}{n}, \\
\left(a_{1} p_{2}\right)^{2} & =\left(a_{1} a_{2}\right)^{2}+\left(a_{2} p_{2}\right)^{2}-2\left(a_{1} a_{2}\right)\left(a_{2} p_{2}\right) \cos \Varangle a_{1} a_{2} p_{2} \\
& =l_{n}^{2}\left[1+4 \sin ^{2}\left(60^{\circ}+\frac{180^{\circ}}{n}\right)+4 \sin \left(60^{\circ}+\frac{180^{\circ}}{n}\right) \sin \frac{540^{\circ}}{n}\right] .
\end{aligned}
$$

Furthermore,

$$
\sin \Varangle a_{2} p_{2} a_{1}=\frac{l_{n}}{\left(a_{1} p_{2}\right)} \sin \Varangle a_{1} a_{2} p_{2}=\frac{l_{n}}{\left(a_{1} p_{2}\right)} \cos \frac{540^{\circ}}{n} .
$$

Hence

$$
\begin{aligned}
\Varangle o a_{1} p_{3} & =\left(90^{\circ}-\frac{180^{\circ}}{n}\right)+60^{\circ}+\Varangle a_{2} a_{1} p_{2} \\
& =150^{\circ}-\frac{180^{\circ}}{n}+\left(180^{\circ}-\left(90^{\circ}+\frac{500^{\circ}}{n}\right)-\Varangle a_{2} p_{2} a_{1}\right) \\
& =240^{\circ}-\frac{720^{\circ}}{n}-\Varangle a_{2} p_{2} a_{1} .
\end{aligned}
$$

It follows

$$
\left(\frac{\left(p_{3} o\right)}{l_{n}}\right)^{2}=\left(\frac{1}{l_{n}}\right)^{2}+\frac{\left(a_{1} p_{2}\right)^{2}}{l_{n}^{2}}+2 \cdot \frac{1}{l_{n}} \cdot \frac{\left(a_{1} p_{2}\right)}{l_{n}} \cos \left(\frac{720^{\circ}}{n}+\Varangle a_{2} p_{2} a_{1}-60^{\circ}\right) .
$$

We compute $\left(\left(p_{3} o\right) / l_{n}\right)^{2}$ for $n=8,9,10$.

\begin{tabular}{rccccc}
\hline$n$ & $\left(a_{1} p_{2}\right)^{2} / l_{n}^{2}$ & $\left(a_{1} p_{2}\right) / l_{n}$ & $\sin 4 a_{2} p_{2} a_{1}$ & $1 / l_{n}$ & $\left(p_{3} o\right)^{2} / l_{n}^{2}$ \\
\hline 8 & $>8.595$ & $>2.937$ & $<0.137$ & $>1.306$ & $>16.3$ \\
9 & $>8.290$ & $>2.879$ & $<0.175$ & $>1.461$ & $>17.7$ \\
10 & $>7.990$ & $>2.826$ & $<0.210$ & $>1.618$ & $>18.9$ \\
\hline
\end{tabular}

\section{Topology 5 (Fig. 5)}

Note that $\triangle p_{1} p_{2} a_{3}=\Delta p_{1} a_{2} a_{4}$. Hence $\left(p_{2} a_{3}\right)=\left(a_{3} a_{1}\right)$. Also note that $\left(p_{1} p_{2}\right)=$ $\left(p_{1} a_{4}\right)=\left(p_{1} a_{1}\right)$. Hence $\triangle p_{1} a_{3} p_{2}=\Delta a_{1} a_{3} p_{1}$. It follows

$$
\varangle p_{1} a_{3} p_{2}=\Varangle a_{1} a_{3} p_{1}=60^{\circ}+180^{\circ} / n
$$

and

$$
\Varangle a_{1} a_{3} p_{2}=120^{\circ}+360^{\circ} / n \text {. }
$$




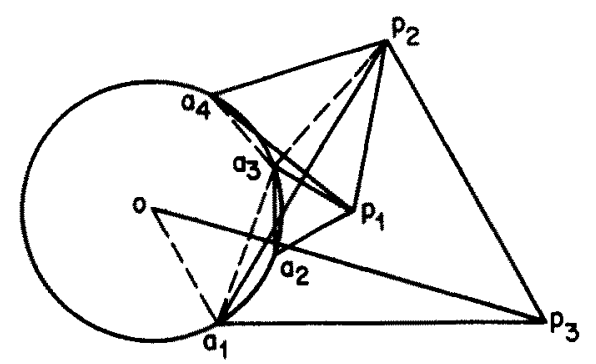

Fig. 5. Melzak's construction for topology 5.

Furthermore,

$$
\begin{aligned}
\left(p_{2} a_{3}\right) & =\left(a_{2} a_{4}\right)=\left(a_{1} a_{3}\right)=2 l_{n}\left[\frac{1-\cos \left(180^{\circ}(n-2) / n\right)}{2}\right]^{1 / 2} \\
& =2 l_{n} \sin \left(180^{\circ}-\frac{180^{\circ}}{n}\right)=2 l_{n} \cos \frac{180^{\circ}}{n}
\end{aligned}
$$

Therefore,

$$
\begin{aligned}
& \left(p_{3} a_{1}\right)=\left(a_{1} p_{2}\right)=\left[\left(a_{1} a_{3}\right)^{2}+\left(a_{3} p_{2}\right)^{2}-2\left(a_{1} a_{3}\right)\left(a_{3} p_{2}\right) \cos \Varangle a_{1} a_{3} p_{2}\right]^{1 / 2} \\
& =4 l_{n} \cos \frac{180^{\circ}}{n}\left[\frac{1-\cos \left(120^{\circ}+360^{\circ} / n\right)}{2}\right]^{1 / 2} \\
& =4 l_{n} \cos \frac{180^{\circ}}{n} \sin \left(60^{\circ}+\frac{180^{\circ}}{n}\right) \\
& =2 l_{n}\left[\sin \left(60^{\circ}+\frac{360^{\circ}}{n}\right)+\sin 60^{\circ}\right] \quad \text { using } 2 \sin A \cos B \\
& =\sin (A+B)+\sin (A-B) \\
& \geq 2 l_{n}\left[\sin \left(60^{\circ}+\frac{360^{\circ}}{8}\right)+\sin 60^{\circ}\right]>3.663 l_{n} \quad \text { for } n \geq 8 \text {. }
\end{aligned}
$$

Finally

$$
\begin{aligned}
\varangle o a_{1} p_{3} & =60^{\circ}+\left(90^{\circ}-\frac{360^{\circ}}{n}\right)-90^{\circ}-\left(60^{\circ}+\frac{160^{\circ}}{n}\right)=180^{\circ}-\frac{540^{\circ}}{n}, \\
\frac{\left(p_{3} o\right)^{2}}{l_{n}^{2}} & =\frac{\left(a_{1} o\right)^{2}}{l_{n}^{2}}+\frac{\left(p_{3} a_{1}\right)^{2}}{l_{n}^{2}}+\frac{\left(p_{3} a_{1}\right)^{2}}{l_{n}^{2}}-2 \frac{\left(a_{1} o\right)}{l_{n}} \frac{\left(p_{2} a_{1}\right)}{l_{n}} \cos \Varangle o a_{1} p_{3} \\
& >\frac{1}{l_{8}^{2}+}(3.663)^{2}+2 \frac{1}{l_{8}}(3.663) \cos \frac{540^{\circ}}{8} \\
& =(1.306)^{2}+(3.063)^{2}+2(1.306)(3.663)(0.382) \\
& =18.778>16 .
\end{aligned}
$$


Topology 6 (Fig. 6)

$$
\begin{aligned}
\left(p_{1} p_{3}\right)^{2}= & \left(p_{1} p_{2}\right)^{2}=\left(p_{1} o\right)^{2}+\left(p_{2} o\right)^{2}-2\left(p_{1} o\right)\left(p_{2} o\right) \cos \Varangle p_{2} o p_{1} \\
& =2\left(p_{1} o\right)^{2}\left(1-\cos \frac{720^{\circ}}{n}\right) \\
\left(p_{3} o\right)^{2}= & \left(p_{1} o\right)^{2}+\left(p_{1} p_{3}\right)^{2}-\left(p_{1} o\right)\left(p_{1} p_{3}\right) \cos \Varangle o p_{1} p_{3} \\
= & \left(p_{1} o\right)^{2}\left[1+2\left(1-\cos \frac{720^{\circ}}{n}\right)\right. \\
& -2 \sqrt{2\left(1-\cos \frac{720^{\circ}}{n}\right)} \cos \left(60^{\circ}+90^{\circ}-\frac{1}{2} \Varangle p_{3} o p_{1}\right) \\
= & \left(p_{1} o\right)^{2}\left[3-\cos \frac{720^{\circ}}{n}+2 \sqrt{2\left(1-\cos \frac{720^{\circ}}{n}\right)} \cos \left(30^{\circ}+\frac{360^{\circ}}{n}\right)\right] \\
\left(\left(p_{3} o\right) / l_{n}\right)^{2}= & \left(\left(p_{1} o\right) / l_{n}\right)^{2}\left[3-2 \cos \frac{720^{\circ}}{n}+2 \sqrt{2\left(1-\cos \frac{720^{\circ}}{n}\right)} \cos \left(30^{\circ}+\frac{360^{\circ}}{n}\right)\right]
\end{aligned}
$$

We compute $\left(p_{3} o / l_{n}\right)^{2}$ for $n=8,9,10$.

\begin{tabular}{rccc}
\hline$n$ & First term & Second term & Product \\
\hline 8 & $>4.297$ & $>3.732$ & $>16.039$ \\
9 & $>6.536$ & $>3.056$ & $>19.963$ \\
10 & $>8.073$ & $>2.525$ & $>20.384$ \\
\hline
\end{tabular}

We now prove Theorem 1 for $8 \leq n \leq 10$. Suppose that $T$ is an SMT for $A_{n}$ with a Steiner point. Since $q$ can be either endpoint of $e$, we may assume that the number of regular points in $T_{1}$ does not exceed that of $T_{2}$, i.e., $k \leq n / 2$. For $n=8$ and 9 Lemmas $8-11$ say that $T_{1}$ is not optimal. For $n=10$ the only case that needs to be considered is when $T_{1}$ and $T_{2}$ cover five regular points each.

Consider the two Steiner paths $P_{1}$ and $P_{2}$ containing $e$. We may assume without loss of generality that $a_{1}$ and $a_{10}$ are the endpoints for $P_{1}$, while $a_{5}$ and $a_{6}$ are the endpoints of $P_{2}$. Let $m_{1}$ and $m_{2}$ denote the number of Steiner points on $P_{1}$ and $P_{2}$. By Lemma $5 m_{1}, m_{2} \leq 4$. Since

$$
\left(a_{1} a_{5}\right)=\sqrt{2\left(1-\cos 144^{\circ}\right)}>1.9>3 l_{10}
$$

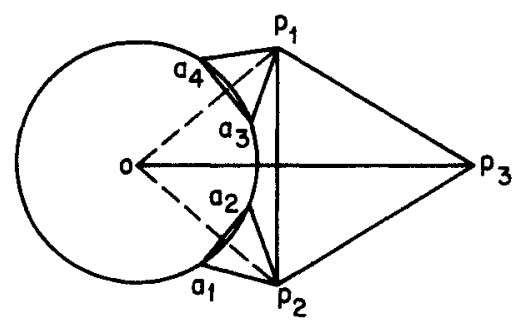

Fig. 6. Melzak's construction for topology 6 . 


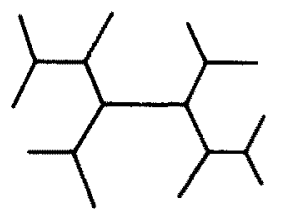

(a)

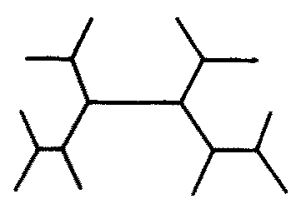

(b)

Fig. 7. Two topologies for $m_{1}=m_{2}=4$.

and each edge in $T$ is shorter than $l_{10}$, there must be at least four edges connecting $a_{1}$ and $a_{5}$. Therefore $m_{1}=m_{2}=4$.

There exist two nonisomorphic topologies for $m_{1}=m_{2}=4$ as shown in Fig. 7 . If $T$ has topology 7(a), then by Lemma $6 T$ must be asymmetric with respect to the center which is on $e$. Therefore we can turn the left half of the tree upside down and obtain a tree of the same length but having 7(b) as its topology (Fig. 8). Namely, it suffices to prove that $T$ cannot have $7(b)$ as its topology.

In $\triangle s_{4} a_{4} a_{5}, 4 s_{4} a_{4} a_{5}=\frac{2}{10} \cdot 180^{\circ}=36^{\circ}$ :

$$
\left(a_{5} a_{4}\right)=\frac{\left(a_{4} a_{5}\right) \sin 36^{\circ}}{\sin 120^{\circ}}=\frac{(0.618)(0.588)}{0.866}=0.42
$$

Extend $\left[a_{5}, s_{4}\right]$ and $\left[a_{1}, s_{1}\right]$ to meet at $b$. Then $b s_{1} s_{3} s_{4}$ is a parallelogram. Hence $\left(s_{4} b\right)=\left(s_{3} s_{1}\right)$ and $\left(s_{1} b\right)=\left(s_{3} s_{4}\right)$. In $\triangle b a_{5} a_{1}, \Varangle b a_{5} a_{1}=\Varangle a_{5} a_{1} b=30$ and $\Varangle a_{1} b a_{5}=$ 120. Furthermore,

$$
\left(a_{1} b\right)=\left(a_{5} b\right)=\left(a_{5} s_{4}\right)+\left(s_{3} s_{1}\right) \leq 0.4204+0.618=1.038
$$

Therefore

$$
\left(a_{1} a_{5}\right)=\sqrt{3} a_{1} b \leq(1.732)(1.038)=1.798
$$

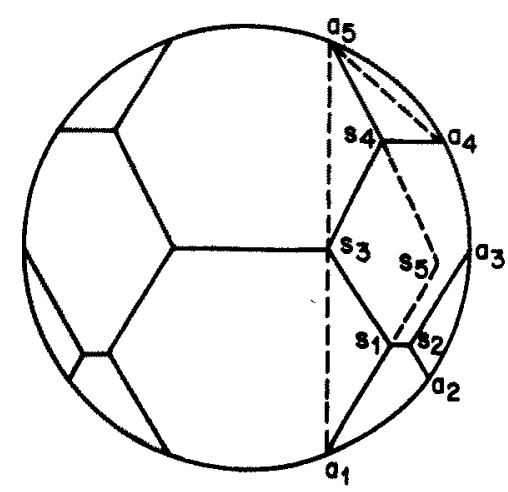

Flg. 8. A tree for topology 7(b). 


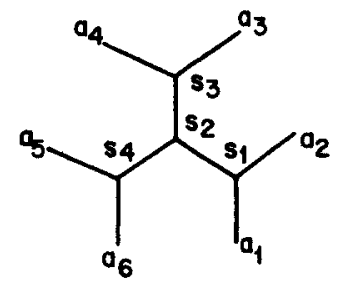

Fig. 9. A unique topology for $n=6$.

But from $\triangle a_{1} a_{5} o$

$$
\left(a_{1} a_{5}\right)=\sqrt{2\left(1-\cos 144^{\circ}\right)}>1.9, \quad \text { a contradiction. }
$$

\section{Proof of Theorem 1 for $n=6,7$}

For $n=6$, Lemma 4 reduces the nonisomorphic topologies to the unique one shown in Fig. 9. Since this topology is symmetric with respect to $s_{2}, s_{2}$ must be the center $o$ and $T$ must be symmetric with respect to $o$. Therefore the length of $T$ is $3 \sqrt{3}>5$ which is the length of an MST.

For $n=7$ Lemma 4 reduces the nonisomorphic topologies to the three shown in Fig. 10. Topology 10(a) can be quickly disposed of by comparing the angles of the polygonal path $a_{1} a_{2} a_{3} a_{4} a_{5} a_{7}$ and those of the Steiner path $a_{1} s_{1} s_{2} s_{3} s_{4} a_{7}$, using Lemma 3 . The length of the tree yielded by topology $10(\mathrm{c})$ has been computed in [13] to be 5.6676>5.2068 which is the length of an MST. We now show that the tree yielded by topology $10(\mathrm{~b})$ is not an SMT as $\left(a_{4} s_{3}\right)>l_{7}$.

Since the topology $10(b)$ is symmetric with respect to $\left[a_{4}, s_{3}\right], T$ must be symmetric to $D$ and $\left[a_{4}, s_{3}\right]$ must overlap with $D$ (see Fig. 11) Extend $\left[a_{3}, s_{2}\right]$ to $b$ such that $\left[a_{1}, b\right] \|\left[s_{1}, s_{2}\right]$. Extend $\left[a_{4}, s_{3}\right]$ to $c$ such that $\left[a_{1}, c\right] \|\left[s_{1}, s_{3}\right]$. Then

$$
\left(a_{4} s_{3}\right)=\left(a_{4} c\right)-\left(a_{3} b\right)+\left(a_{3} s_{2}\right) .
$$

Now

$$
\Varangle a_{4} a_{1} c=\Varangle a_{3} a_{2} s_{2}=\Varangle a_{3} a_{2} a_{6}-\Varangle s_{2} a_{2} a_{6}=\frac{3}{7} \cdot 180^{\circ}-30^{\circ}=\frac{330^{\circ}}{7} .
$$

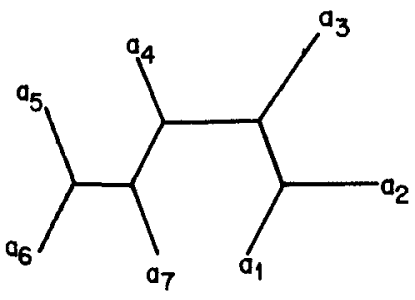

(a)

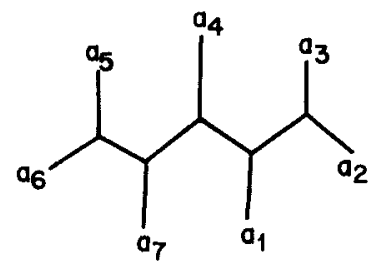

(b)

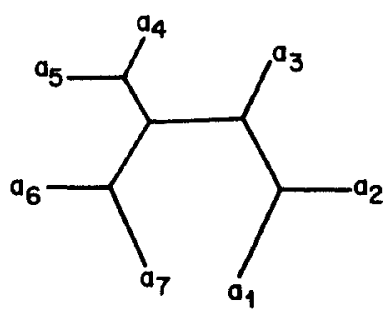

(c)

Fig. 10. Three topologies for $n=7$. 


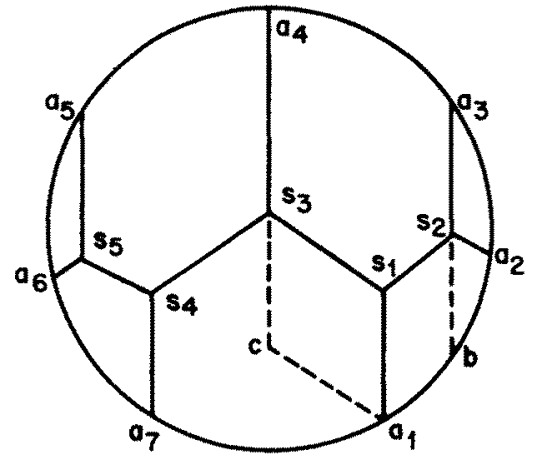

Fig. 11. Tree for 10(b).

Hence

$$
\begin{aligned}
& \left(a_{3} s_{2}\right)=\frac{\left(a_{2} a_{3}\right) \sin \left(330^{\circ} / 7\right)}{\sin 120^{\circ}}=\frac{\sqrt{2\left[1-\cos \left(360^{\circ} / 7\right)\right]} \sin \left(330^{\circ} / 7\right)}{\sin 120^{\circ}} \\
& \left(a_{4} c\right)=\frac{\left(a_{1} a_{4}\right) \sin \left(330^{\circ} / 7\right)}{\sin 120^{\circ}}=\frac{\sqrt{2\left[1-\cos \left(1080^{\circ} / 7\right)\right]} \sin \left(330^{\circ} / 7\right)}{\sin 120^{\circ}} .
\end{aligned}
$$

Furthermore,

$$
\Varangle b a_{1} a_{3}=\Varangle s_{5} a_{6} a_{5}=\chi_{4} a_{3} a_{2} s_{2} \quad \text { by symmetry. }
$$

Hence

$$
\left(a_{3} b\right)=\frac{\left(a_{1} a_{3}\right) \sin \left(330^{\circ} / 7\right)}{\sin 120^{\circ}}=\frac{\sqrt{\left.2\left[1-\cos \left(720^{\circ}\right) / 7\right)\right]} \sin \left(330^{\circ} / 7\right)}{\sin 120^{\circ}} .
$$

Therefore,

$$
\begin{aligned}
\left(a_{4} s_{3}\right)= & {\left[\sqrt{2\left(1-\cos \frac{360^{\circ}}{7}\right)}+\sqrt{2\left(1-\cos \frac{1080^{\circ}}{7}\right)}\right.} \\
& \left.-\sqrt{2\left(1-\cos \frac{720^{\circ}}{7}\right)}\right] \frac{\sin \left(330^{\circ} / 7\right)}{\sin 120^{\circ}} \\
= & (1.950+0.868-1.563)(0.733) / 0.866=1.06>l_{7} .
\end{aligned}
$$

\section{The Longest Steiner Minimal Trees for $\boldsymbol{n}$ Cocircular Points}

The MST for any $n$ cocircular points is clearly longest when the $n$ points are equally spaced. Now for any $n$ given points, the length of an SMT never exceeds that of an MST. Furthermore, Theorem 1 tells us that an MST is an SMT for the equally spaced set if $n \geq 6$. Therefore Theorem 2 is proved for $n \geq 6$. The proof of Theorem 2 for $n=3,4,5$ will each be given separately.

Let $C_{n}$ denote a set of $n$ points on the unit circle. Let $P_{n}$ denote the enclosing polygon of $C_{n}$. 
Lemma 12. For $3 \leq n \leq 5$, if one of the angles of $P_{n}$ is $120^{\circ}$ or larger, than an $S M T$ for $C_{n}$ is shorter than that for $A_{n}$.

Proof. We show that an MST for $C_{n}$ is shorter than the SMT for $A_{n}$.

Without loss of generality, assume $\Varangle a_{1} a_{2} a_{3} \geq 120^{\circ}$. Then

$$
\Varangle a_{1} o a_{2}+\Varangle a_{2} o a_{3} \leq 120^{\circ} \text {. }
$$

By standard minimization techniques it is easily seen that the longest MST for $n$ cocircular points satisfies the angle conditions

$$
\Varangle a_{1} o a_{2}=\Varangle a_{2} o a_{3}=60^{\circ} \text {, }
$$

and

$$
\Varangle a_{3} o a_{4}=\cdots=\Varangle a_{n} o a_{1}=240^{\circ} /(n-2) \text {. }
$$

The length of such an MST is

$$
\begin{aligned}
2 \sqrt{2\left(1-\cos 60^{\circ}\right)}+(n-3) \sqrt{2\left[1-\cos \left(\frac{240^{\circ}}{n-2}\right)\right]} & \\
= & \left\{\begin{array}{lll}
2<3 & \text { for } & n=3, \\
2+\sqrt{3}<\sqrt{2}+\sqrt{6} & \text { for } & n=4, \\
2+2.572<4.574 & \text { for } & n=5,
\end{array}\right.
\end{aligned}
$$

where the right side of the inequality is the length of an SMT for $A_{n}$.

Corollary. If an SMT for $C_{n}$ is not full, then its length is shorter than that of $A_{n}$.

We now prove Theorem 2 for $n=3$. Consider $C_{3}$ such that all angles of $P_{3}$ are less than 120. Construct a regular $\triangle B C D$ such that $A$ and $D$ are on different sides of $[B, C]$. Then $(A D)$ is the length of the SMT for $C_{3}$ (see Fig. 12). Let $\Varangle O B D=\theta$ :

$$
\begin{aligned}
(A D) & \leq(A O)+(O D) \\
& =1+\frac{(O B) \sin \theta}{\sin 30} \leq 3
\end{aligned}
$$

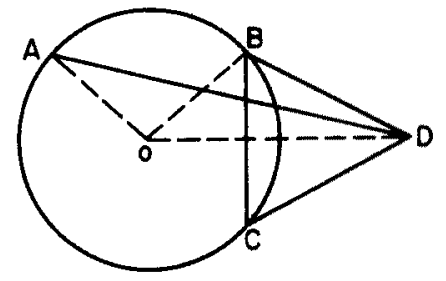

Fig. 12. A Steiner tree for $n=3$. 


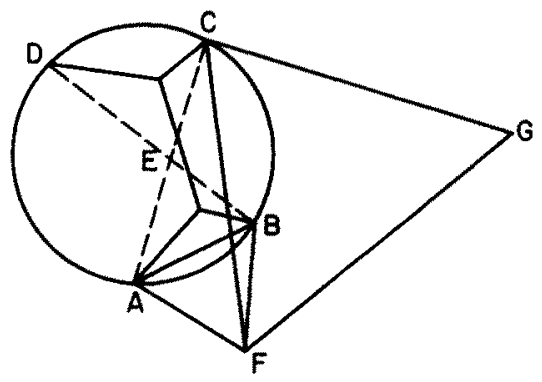

Fig. 13. A Steiner tree for $n=4$.

Next we prove Theorem 2 for $n=4$. Consider $C_{4}$ such that all angles of $P_{4}$ are less than $120^{\circ}$. Suppose that the diagonals $[A, C]$ and $[B, D]$ meet at $E$. Without loss of generality, assume that $\Varangle A E B \leq 90$. Then the Steiner tree $T$ as shown in Fig. 13 exists.

Construct a regular $\triangle A B F$ and a regular $\triangle F C G$. Then the length of $T$ is $(D G)$. But $\triangle G F B \equiv \triangle C F A$, hence $(G B)=(A C)$ and $\Varangle F B G=\Varangle F A C$. Furthermore,

$$
\begin{aligned}
\Varangle G B D & =360^{\circ}-\not G B F-\measuredangle D B F \\
& =360^{\circ}-\measuredangle F A C-\measuredangle E B F \\
& =360^{\circ}-\left(360^{\circ}-60^{\circ}-\not \Varangle A E B\right) \\
& =60^{\circ}+\not A E B .
\end{aligned}
$$

In $\triangle G D B$

$$
\begin{aligned}
(G D)^{2} & =\left[(G B)^{2}+(B D)^{2}-2(G B)(B D) \cos 4 G B D\right]^{1 / 2} \\
& =\left[(A C)^{2}+(B D)^{2}-2(A C)(B D) \cos (60+\Varangle A E B)\right]^{1 / 2} \\
& \leq\left(2^{2}+2^{2}-2 \cdot 2 \cdot 2 \cdot \cos 150^{\circ}\right)^{1 / 2} \\
& =(8+4 \sqrt{3})^{1 / 2} \\
& <\sqrt{2}+\sqrt{6} .
\end{aligned}
$$

Finally, we prove Theorem 2 for $n=5$. Without loss of generality, assume that the polygons under study are inscribed in a unit circle. Let $M$ denote the length of an SMT for $A_{5}$. Then it is straightforward to calculate

$$
\begin{aligned}
M & =4\left(\sin 36^{\circ}+\sin 72^{\circ}\right) \sin 96^{\circ} \\
& =4.574 .
\end{aligned}
$$

Consider a $C_{5}$ with points $A, B, C, D$, and $E$. By Lemma 12 we may assume that $\Varangle A, \Varangle B, \Varangle C, \Varangle D$, and $\Varangle E$ are all less than $120^{\circ}$. Therefore there exist five full Steiner trees where one of them is as shown in Fig. 14 and the other other four can be obtained by rotating the points. 


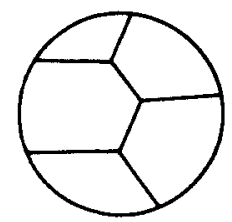

Fig. 14. A Steiner tree for $n=5$.

Let $M_{1}, M_{2}, M_{3}, M_{4}$, and $M_{5}$ denote the lengths of these five trees, respectively. We prove that

$$
\sum_{i=1}^{5} M_{i}<5 M
$$

where $M$ is the length of an SMT for $A_{5}$. Therefore the SMT for $C_{5}$, which is the shortest one among the five trees, must be shorter than $M$.

Construct equilateral triangles $\triangle A B D^{\prime}, \triangle B C E^{\prime}, \triangle C D A^{\prime}, \triangle D E B^{\prime}, \triangle E A C^{\prime}$, $\triangle A^{\prime} C^{\prime} B^{\prime \prime}, \triangle B^{\prime} D^{\prime} C^{\prime \prime}, \triangle C^{\prime} E^{\prime} D^{\prime \prime}, \triangle D^{\prime} A^{\prime} E^{\prime \prime}$, and $\triangle E^{\prime} B^{\prime} A^{\prime \prime}$ (see Fig. 15). Then $\left[A, A^{\prime \prime}\right],\left[B, B^{\prime \prime}\right],\left[C, C^{\prime \prime}\right],\left[D, D^{\prime \prime}\right]$, and $\left[E, E^{\prime \prime}\right]$ are the five axes. Since $\left(x^{\prime \prime}, x\right) \leq$ $\left(x^{\prime \prime}, o\right)+(o, x)=\left(x^{\prime \prime}, o\right)+1$ for $x=A, B, C, D, E$, it suffices to prove

$$
S \equiv\left(A^{\prime \prime} o\right)+\left(B^{\prime \prime} o\right)+\left(C^{\prime \prime} o\right)+\left(D^{\prime \prime} o\right)+\left(E^{\prime \prime} o\right) \leq 5(M-1)
$$

Construct a circle through the three points $A^{\prime \prime}, B^{\prime}$, and $E^{\prime}$ and meet $\left[A^{\prime \prime}, o\right]$ (or its extension) at $G$. Then

$$
\left(A^{\prime \prime} o\right)=\left(G B^{\prime}\right)+\left(G E^{\prime}\right)+(G o) \quad(\text { or }-(G o)) .
$$

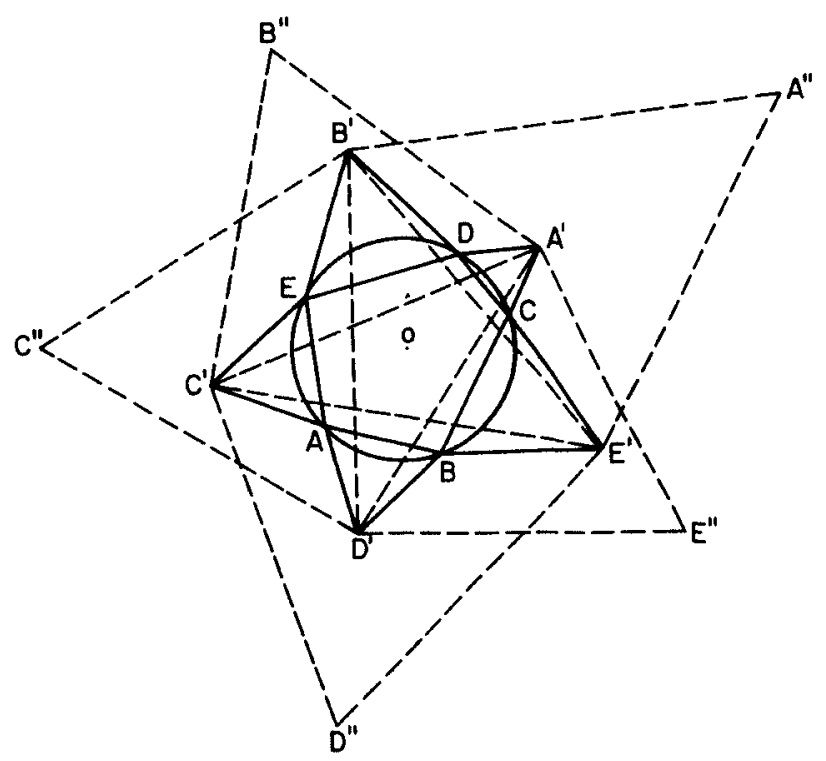

Fig. 15. Axes for the five full Steiner trees. 
Define

$$
\begin{aligned}
& \Varangle A^{\prime \prime} \circ B^{\prime}=\alpha_{1}, \quad \Varangle B^{\prime \prime} o C^{\prime}=\alpha_{2}, \ldots, \quad \Varangle E^{\prime \prime} o A^{\prime}=\alpha_{5} \text {, } \\
& \Varangle E^{\prime} \circ A^{\prime \prime}=\beta_{1}, \quad \Varangle A^{\prime} \circ B^{\prime \prime}=\beta_{2}, \ldots, \quad \Varangle D^{\prime} o E^{\prime \prime}=\beta_{5}, \\
& \Varangle C o D=2 \theta_{1}, \quad \Varangle D o E=2 \theta_{2}, \ldots, \quad \Varangle B o C=2 \theta_{5} \text {. }
\end{aligned}
$$

Since

$$
\Varangle A^{\prime \prime} G B^{\prime}=\frac{1}{2} \Varangle E^{\prime} G B^{\prime}=\frac{1}{2}\left(180^{\circ}-\not B^{\prime} A^{\prime \prime} E^{\prime}\right)=60^{\circ},
$$

we have

$$
\left(A^{\prime \prime} o\right)=\frac{\sin \alpha_{1}}{\sin 60^{\circ}}\left(o B^{\prime}\right)+\frac{\sin \beta_{1}}{\sin 60^{\circ}}\left(o E^{\prime}\right)+\frac{\sin \left(60^{\circ}-\alpha_{1}\right)}{\sin 60^{\circ}}\left(o B^{\prime}\right)
$$

Note that

$$
\begin{gathered}
\left(o B^{\prime}\right)=2 \sin \left(30^{\circ}+\theta_{2}\right), \\
\left(o E^{\prime}\right)=2 \sin \left(30^{\circ}+\theta_{5}\right) \\
\frac{\sin \left(60^{\circ}-\alpha_{1}\right)}{\sin 60^{\circ}}\left(o B^{\prime}\right)=\frac{\sin \left(60^{\circ}-\beta_{1}\right)}{\sin 60^{\circ}}\left(o E^{\prime}\right) .
\end{gathered}
$$

we have

$$
\begin{aligned}
\left(A^{\prime \prime} o\right)= & \frac{1}{\sqrt{3}}\left\{\left[\left(o B^{\prime}\right) \sin \alpha_{1}+\left(o E^{\prime}\right) \sin \beta_{1}\right]\right. \\
& \left.+\left(o B^{\prime}\right)\left[\sin \alpha_{1}+\sin \left(60^{\circ}-\alpha_{1}\right)\right]+\left(o E^{\prime}\right)\left[\sin \beta_{1}+\sin \left(60^{\circ}-\beta_{1}\right)\right]\right\} \\
= & \frac{1}{\sqrt{3}}\left\{\left[\left(o B^{\prime}\right) \sin \alpha_{1}+\left(o E^{\prime}\right) \sin \beta_{1}\right]\right. \\
& \left.\times\left(o B^{\prime}\right) \cos \left(30^{\circ}-\alpha_{1}\right)+\left(o E^{\prime}\right) \cos \left(30^{\circ}-\beta_{1}\right)\right\} \\
= & \frac{1}{\sqrt{3}}\left\{\left(o B^{\prime}\right)\left[\sin \alpha_{1}+\cos \left(30^{\circ}-\alpha_{1}\right)\right]+\left(o E^{\prime}\right)\left[\sin \beta_{1}+\cos \left(30^{\circ}-\beta_{1}\right)\right]\right\} \\
= & \frac{1}{\sqrt{3}}\left\{\left(o B^{\prime}\right)\left[\sin \alpha_{1}+\sin \left(60^{\circ}+\alpha_{1}\right)\right]+\left(o E^{\prime}\right)\left[\sin \beta_{1}+\sin \left(60^{\circ}+\beta_{1}\right)\right]\right\} \\
= & \frac{1}{\sqrt{3}}\left\{\left(o B^{\prime}\right) \sin \left(30^{\circ}+\alpha_{1}\right) \cos 30^{\circ}+\left(o E^{\prime}\right) \sin \left(30^{\circ}+\beta_{1}\right) \cos 30^{\circ}\right\} \\
= & \sin \left(30^{\circ}+\alpha_{1}\right) \sin \left(30^{\circ}+\theta_{2}\right)+\sin \left(30^{\circ}+\beta_{1}\right) \sin \left(30^{\circ}+\theta_{5}\right) \\
= & \cos \left(\alpha_{1}-\theta_{2}\right)-\cos \left(60+\alpha_{1}+\theta_{2}\right)+\cos \left(\beta_{1}-\theta_{5}\right)-\cos \left(60+\beta_{1}+\theta_{5}\right) \\
= & \cos \left(\alpha_{1}-\theta_{2}\right)+\sin \left(\alpha_{1}+\theta_{2}-30^{\circ}\right)+\cos \left(\beta_{1}-\theta_{5}\right)+\sin \left(\beta_{1}+\theta_{5}-30^{\circ}\right) .
\end{aligned}
$$


Therefore we can write

$$
S=S^{\prime}+S^{\prime \prime}
$$

where

$$
\begin{aligned}
S^{\prime}= & \cos \left(\alpha_{1}-\theta_{2}\right)+\cos \left(\beta_{1}-\theta_{5}\right)+\cdots+\cos \left(\alpha_{5}-\theta_{1}\right)+\cos \left(\beta_{5}-\theta_{4}\right), \\
S^{\prime \prime}= & \sin \left(\alpha_{1}+\theta_{2}-30^{\circ}\right)+\sin \left(\beta_{1}+\theta_{5}-30^{\circ}\right)+\cdots+\sin \left(\alpha_{5}+\theta_{1}-30^{\circ}\right) \\
& +\cos \left(\beta_{5}+\theta_{4}-30^{\circ}\right) .
\end{aligned}
$$

To bound $S^{\prime}$ and $S^{\prime \prime}$ we need the following lemma.

Lemma 13. Let $\Varangle X Y Z=y$ where $60^{\circ}<y<180^{\circ}$. Construct equilateral triangle $\triangle X Z W$ and define $\triangle W Y Z=W$ (see Fig. 16). Then

$$
\min \left\{60^{\circ}, y-60^{\circ}\right\} \leq w \leq \max \left\{60^{\circ}, y-60^{\circ}\right\} \text {. }
$$

Proof. Construct a circle circumscribing the three points $X, Y$, and $Z$. Then $W$ lies outside of the circle if $y<120^{\circ}$, on the circle if $y=120^{\circ}$, and inside the circle if $y>120^{\circ}$. Consider the first case. When $Y$ moves from $Z$ to $X$ along the arc $Z X$, clearly, $w$ increases from $y-60^{\circ}$ to $60^{\circ}$ since the angle of the arc it faces also increases. An analogous argument proves Lemma 13 for the other two cases.

We may assume without loss of generality that $\theta_{i} \leq 41.25^{\circ}$ for $1 \leq i \leq 5$ since otherwise the MST for $C_{5}$ is already shorter than $M$.

Define $\theta_{6}=\theta_{1}$ and $\theta_{0}=\theta_{5}$. By Lemma 13

$$
\alpha_{i} \geq \min \left\{60^{\circ}, \theta_{i-1}+2 \theta_{i}+\theta_{i+1}-60^{\circ}\right\}>\theta_{i+1} \text {. }
$$

Furthermore, $\alpha_{i}-\theta_{i+1} \leq \max \left\{60^{\circ}, \theta_{i-1}+2 \theta_{i}+\theta_{i+1}-60^{\circ}\right\}-\theta_{i+1} \leq 63.75^{\circ}$. Similarly, we can show $\theta<\beta_{i}-\theta_{i-1} \leq 63.75^{\circ}$. Since $\cos x$ is concave for $0^{\circ} \leq x \leq 90^{\circ}$ and

$$
\sum_{i=1}^{4}\left(\alpha_{i}-\theta_{i+1}\right)+\alpha_{5}-\theta_{1}+\beta_{1}-\theta_{5}+\sum_{i=2}^{5}\left(\beta_{i}-\theta_{i-1}\right)=360^{\circ}
$$

$S^{\prime}$ achieves its maximum when

$$
\alpha_{1}-\theta_{2}=a_{2}-\theta_{3}=\cdots=\alpha_{5}-\theta_{1}=\beta_{1}-\theta_{5}=\cdots=\beta_{5}-\theta_{1}=360^{\circ} / 10=36^{\circ} \text {. }
$$
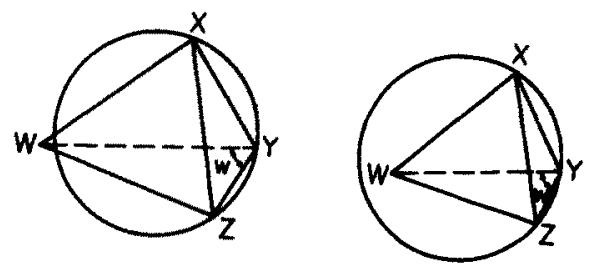

Fig. 16. The range of angle $w$. 
Next note that $\theta_{1}+\theta_{2}=180^{\circ}-\measuredangle C D E>60^{\circ}$ and $\theta_{1} \leq 41.25^{\circ}$ implies $\theta_{2}>15^{\circ}$. Hence

$$
\begin{aligned}
0^{\circ} & \leq \alpha_{i}+\theta_{i+1}-30^{\circ} \leq \max \left\{30^{\circ}+\theta_{i+1}, \theta_{i-1}+2 \theta_{i}+2 \theta_{i+1}-90^{\circ}\right\} \\
& \leq \max \left\{71.25^{\circ}, 113.75^{\circ}\right\} .
\end{aligned}
$$

Since $\sin x$ is concave for $0^{\circ} \leq x \leq 180^{\circ}$ and

$$
\alpha_{1}+\theta_{2}-30^{\circ}+\beta_{1}+\theta_{5}-30^{\circ}+\cdots+\alpha_{5}+\theta_{1}-30^{\circ}+\beta_{5}+\theta_{4}-30^{\circ}=780^{\circ},
$$

$S^{\prime \prime}$ achieves its maximum when

$$
\alpha_{1}+\theta_{2}-30^{\circ}=\beta_{1}+\theta_{5}-30^{\circ}=\cdots=\alpha_{5}+\theta_{1}-30^{\circ}=\beta_{5}+\theta_{4}-30^{\circ}=780^{\circ} / 10=78^{\circ} \text {. }
$$

It is easily verified that when $C_{5}=A_{5}$ the conditions on $\alpha_{i}, \beta_{i}$, and $\theta_{i}$ to maximize $S^{\prime}$ and $S^{\prime \prime}$ are exactly fulfilled and $S=5(M-1)$.

\section{Acknowledgment}

We thank P. Hell for informing us of and summarizing for us the papers of Jarnick and Kössler and of Kotzig.

\section{References}

1. F. R. K. Chung and R. L. Graham, Steiner trees for ladders, Ann. Discrete Math. 2(1978), 173-200.

2. F. R. K. Chung and R. L. Graham, A new bound for euclidean Steiner minimal trees, Ann. N.Y. Acad. Sci. 440 (1985) 328-346.

3. D. Z. Du and F. K. Hwang, A new bound for the Steiner ratio, Trans. Amer. Math. Soc. 228 (1983), 137-148.

4. D. Z. Du and F. K. Hwang, Steiner minimal trees for bar waves, to appear.

5. D. Z. Du, F. K. Hwang, and J. F. Weng, Steiner minimal trees on zig-zag line, Trans. Amer. Math. Soc. 228 (1983), 149-156.

6. D. Z. Du, F. K. Hwang, J. F. Weng, and S. C. Chao, Steiner minimal trees for points on a circle. Proc. Amer. Math. Soc., 95 (1985), 613-618.

7. M. R. Garey, R. L. Graham, and D. S. Johnson, The complexity of computing Steiner minimal trees, SIAM J. Appl. Math. 32 (1977), 835-859.

8. E. N. Gilbert and H. O. Pollak, Steiner minimal trees, SIAM J. Appl. Math. 16 (1968), 1-29.

9. F. K. Hwang, J. F. Weng, and D. Z. Du, A class of full Steiner minimal trees, Discrete Math. 45 (1983), 107-112.

10. V. Jarnik and M. Kössler, O minimálnich grafech obbsahujicich n daných bodu, Casopis Pěst. Mat. Fys. 63 (1934), 223-235.

11. A. Kotzig, Optimálne spojovacie systémy, in Mathematické metódy V Hospodárskej Praxi (V. Kapitoly, ed.), Vydavel'stuo Solvenskej Akadémie vied, Bratislava, 1961.

12. Z. A. Melak, On the problem of Steiner, Canad. Math. Bull 4 (1960), 143-148

13. J. F. Weng and F. K. Hwang, Hexagonal coordinate system and Steiner minimal trees, Discrete Math, to appear.

Received August 1, 1985, and in revised form January 10, 1986. 